\title{
The Influence of Prenatal Trauma, Stress, Social Support, and Years of Residency in the US on Postpartum Maternal Health Status Among Low-Income Latinas
}

\author{
Lekeisha A. Sumner • Jeanette Valentine - David Eisenman • \\ Sawsann Ahmed · Hector Myers · Gail Wyatt . \\ Honghu Liu $\cdot$ Muyu Zhang $\cdot$ Michael A. Rodriguez
}

Published online: 21 July 2010

(C) The Author(s) 2010. This article is published with open access at Springerlink.com

\begin{abstract}
This study examined the associations of prenatal psychosocial factors, including depressive symptoms, post-traumatic stress disorder symptoms, trauma exposure including intimate partner violence, perceived stress, and social support, with perceived postpartum health status. Low-income Latinas $(N=203)$ were recruited from two health plans within the first 12 weeks of their pregnancies and followed through 3 months after birth. Participants completed semi-structured interviews conducted in English or Spanish within the first 12 weeks of pregnancy, and again at 12 weeks postpartum. Perceived health status was measured by the SF-12. Participants with complete followup data $(n=193)$ were used in data analysis. Women were mostly foreign-born $(75 \%)$ with low-incomes $(59 \%)$ and reported postpartum health status in the average range
\end{abstract}

L. A. Sumner $(\square) \cdot$ G. Wyatt · M. Zhang

Department of Psychiatry and Biobehavioral Sciences,

David Geffen School of Medicine at UCLA,

Los Angeles, CA, USA

e-mail: 1sumnerPhd@gmail.com

G. Wyatt

e-mail: gwyatt@mednet.ucla.edu

M. Zhang

e-mail: mzhang@mednet.ucla.edu

J. Valentine

Center for Health Services Research, Semel Research Institute,

David Geffen School of Medicine at UCLA,

Los Angeles, CA, USA

e-mail: jvalentine@pathwaysla.org

D. Eisenman · H. Liu

Division of General Medicine and Health Services Research, Department of Medicine, David Geffen School of Medicine at UCLA, Los Angeles, CA, USA

e-mail: deisenman@mednet.ucla.edu
$(M=102.5 ; \mathrm{SD}=12.2)$. Overall health status was positively associated with decreased levels of perceived stress $(P<.0001)$, being foreign-born and having resided in the US $<10$ years $(P=.003)$. Emotional well-being was positively linked with being foreign-born and having resided in the US $<10$ years $(P=.002)$, increased levels of social support $(P=.01)$, and decreased levels of perceived stress $(P<.001)$. Exposure to non-specific IPV trauma $(P=.01)$ and health problems experienced during pregnancy or delivery $(P=.05)$ were negatively associated with physical health status. Prenatal psychosocial factors and length of residency in the US are differentially predictive of overall postpartum health status and emotional well-being, and have less impact on physical well-being after birth. Health professionals are encouraged to assess these factors in early pregnancy.

H. Liu

e-mail: hhliu@mednet.ucla.edu

S. Ahmed

Department of Psychology, San Diego State University,

San Diego, CA, USA

e-mail: sawssanrahmed@gmail.com

H. Myers

Department of Psychology, UCLA, Los Angeles, CA, USA

e-mail: myers@psych.ucla.edu

M. A. Rodriguez

Department of Family Medicine, David Geffen of Medicine

at UCLA, Los Angeles, CA, USA

e-mail: mrodriguez@mednet.ucla.edu 
Keywords Postpartum maternal health status . Perceived stress - Trauma exposure . US and foreign born Latinas · Low-income

\section{Introduction}

Postpartum health remains a neglected area of study, especially among Latinas who account for $15 \%$ of the U.S. population [1] and approximately $24 \%$ of all US births [2]. The postpartum period, especially 12 weeks postpartum, is a defined period of risk for postpartum depression $[3,4]$ and is often the time when many women return to work $[5,6]$. Consequently, health professionals $[7,8]$ and organizations alike, including the United Nations [9] have called for increased knowledge on how to improve maternal health and well-being. Some evidence illustrates that both socio-demographic and psychosocial factors during pregnancy are associated with maternal health and that low-income women are more likely to report fair-topoor perceived health status compared to those of higher incomes [10-14]. However, most studies that have focused on the influence of depressive symptoms and social support on maternal health status have relied on cross-sectional designs and heterogeneous samples [12-14]. By examining these issues in an understudied population-low income Latinas - this study expands the knowledge base about the possible role of prenatal psychosocial factors during pregnancy in postpartum perceived maternal health status.

Perceived health status has been shown to independently predict objective functioning, disability, mortality and morbidity across a spectrum of populations with diverse incomes and ethnicities [15, 16]. A range of non-medical factors, such as exposure to traumatic events (e.g., intimate partner violence [IPV], childhood trauma) [17, 18] and socio-demographic factors [19] have been shown to contribute to perceived health status. Among pregnant women, health problems experienced during pregnancy or delivery, prenatal depressive symptoms, social support, and stress have been associated with postpartum perceived health status [10-14]. Few studies have examined the contributions of a range of prenatal psychosocial and socio-demographic factors simultaneously with postpartum health status, especially among Latinas for whom the effects of some factors may be particularly pronounced on health status [20]. Studies utilizing a longitudinal design and broader psychosocial context than has been used among pregnant populations will advance what is known about the psychosocial determinants that shape health perceptions, the strength of their contributions, and the effect of these associations on postpartum health status.

This study's purpose was to build upon the prior literature on psychosocial and socio-demographic predictors of perceived postpartum health status by examining the associations of a range of factors during pregnancy with three indices of self-rated maternal health status at 3 months postpartum: overall health, emotional well-being, and physical health. Consistent with prior findings and the tenets of prevailing frameworks of ethnic and immigrant health [20], it was hypothesized that higher levels of overall health, emotional well-being and physical health would be reported by foreign-born women at 12 weeks post-partum who had: higher levels of social support; lower levels of perceived stress; fewer symptoms of depression, PTSD; and no history of exposure to traumatic events (e.g., childhood abuse, lifetime exposure to IPV, exposure to IPV in the previous 12 months). It was also hypothesized that women who experienced few health problems during pregnancy or delivery would report higher levels of physical health status at 12 weeks postpartum.

\section{Methods}

\section{Design and Procedures}

Proyecto Cuna (Baby Cradle) is an on-going longitudinal investigation that began in 2003 to assess the impact of trauma and mental health factors on maternal and child well-being among Latinas, including IPV. Participants were recruited from the obstetrical clinics of two private, nonprofit health plans in Los Angeles County where approximately $80 \%$ of the patient population was Latina. Eligibility criteria included self-report of being at least aged 18 years or older, self-identified as Latina, at least 12 weeks pregnant (confirmed by medical records), not planning to move in the next 12 months, and planning to raise the child themselves (i.e., the child would reside with them and that the participant, with or without the help of a partner would be the child's primary caretaker). Women completed baseline interviews in a private clinic room during the prenatal period and via phone or at home visits at 12 weeks postpartum. Full details of the study recruitment procedures and methodology are published elsewhere [21].

The study protocol was approved by the institutional review board of the University of California, Los Angeles School of Medicine. Between January 2003 and January 2004 , women $(n=1,728)$ were approached by trained bilingual and bicultural research staff and informed about the study while waiting for routine appointments. The research assistants introduced themselves as researchers working on a research study to understand the needs of pregnant Latina women and their babies to help enhance healthcare services provided to them. Because one of the primary objectives of Project CUNA was to examine the 
role of IPV on health, the recruitment goal was to enroll a sample of approximately half of women reporting a lifetime history of IPV (IPV positive) and those reporting no lifetime history of IPV (IPV negative).

Research staff worked closely with clinic personnel to ensure that participants were not approached when their partner or any other person was present. Interested women were screened alone in a private area by the interviewer to determine study eligibility. Women who met the inclusion criteria were screened in a private area by the interviewer to determine study eligibility ( $n=831$ ). Of these 831 women, $83 \%$ agreed to be screened (139 refused to be screened), 46 IPV negative and nine IPV positive women refused to participate, 10 women were lost between eligibility determination and consenting procedures, and 417 IPV negative women were screened after the quota for IPV negative women had already been filled, leaving 210 eligible women who consented to participate. Participants were given the option of choosing to complete the interview in either in English or Spanish. The interview schedule contained 121 mostly closed-ended items. All participants were compensated $\$ 20$ for baseline and $\$ 40$ for follow-up interviews. Of the 210 participants who met eligibility criteria and entered the study during pregnancy, 203 women completed questionnaires in the 3-month postpartum follow-up survey and thus constitute the study sample. Demographic and psychosocial characteristics of the study as measured at prenatal entry are shown in Table 1 . With a mean age of 27.7, the majority of the women in this study were married $(88.7 \%)$, and $59.2 \%$ were from low-income households with a total annual family income of $<\$ 20,000$. Most women in this sample were foreign-born (75.9\%), and completed the semi-structured interviews in Spanish $(61.7 \%)$.

\section{Predictor Variables}

IPV status was assessed during pregnancy using five questions from the Abuse Assessment Screen (AAS), a clinical screen for emotional, physical and/or sexual abuse within the last 12 months, and was developed for use with both pregnant and non-pregnant women [22]. Criterionrelated validity has been established by comparing responses to individual items on the AAS with scores from other violence scales that have demonstrated reliability and validity, and have been used in family violence research. A significant positive relationship $(P<.01)$ has been found between the AAS and other instruments with reliability established at $97.5 \%$ using a test-retest method [22]. Women reporting yes onto any one of the items within 12 months of the current pregnancy were classified as "recent" IPV exposure and were distinguished from women reporting "remote" exposure to IPV. Women who
Table 1 Demographic and psychosocial characteristics of study sample $(n=203)$

\begin{tabular}{|c|c|}
\hline Variable & $m(\mathrm{SD})$ or $\%(n)$ \\
\hline Age & $27.7(\mathrm{SD}=5.7)$ \\
\hline Income $(\leq \$ 20,000)$ & $59.2 \%$ \\
\hline Poverty index* & $1.3(\mathrm{SD}=1.4)$ \\
\hline Employed (full or part-time) & $44.4 \%(88)$ \\
\hline Education $<\mathrm{HS}$ & $45.5 \%(88)$ \\
\hline Married & $88.7 \%(180)$ \\
\hline \multicolumn{2}{|l|}{ Nativity status } \\
\hline US-born & $24.2 \%(49)$ \\
\hline Non-US born $\leq 10$ yrs in US & $36.9 \%(75)$ \\
\hline Non-US-born $\geq 10$ yrs in US & $38.9 \%(79)$ \\
\hline Language of interview-Spanish & $61.7 \%(122)$ \\
\hline \multicolumn{2}{|l|}{ IPV status } \\
\hline Recent (current/within previous $12 \mathrm{mos}$ ) & $21.7 \%(44)$ \\
\hline Remote (previous $>12$ mos) & $22.6 \%(46)$ \\
\hline None & $55.7 \%(113)$ \\
\hline \# Non-IPV trauma events $(0-7)$ & $1.15(\mathrm{SD}=1.59)$ \\
\hline Perceived stress score & $8.10(\mathrm{SD}=3.50)$ \\
\hline Depression score & $2.31(\mathrm{SD}=3.67)$ \\
\hline PTSD score & $25.39(\mathrm{SD}=13.68)$ \\
\hline Social support score & $37.17(\mathrm{SD}=7.31)$ \\
\hline \# Pregnancy/labor/delivery problems & $1.03(\mathrm{SD}=1.06)$ \\
\hline Labor pain $>3$ weeks prior to delivery & $8.1 \%(57)$ \\
\hline High blood pressure & $10.8 \%(22)$ \\
\hline Diabetes & $24.6 \%(50)$ \\
\hline Urinary tract infection & $12.8 \%(26)$ \\
\hline Sexually transmitted infection & $3 \%(6)$ \\
\hline Anemia & $24.0 \%(49)$ \\
\hline Non-vaginal delivery & $24.3 \%(49)$ \\
\hline
\end{tabular}

* Poverty index is the total family income divided by the federal poverty guidelines for the specified number of persons in each household. With an index score of " 1 " the family is considered to be living at the poverty level. A score above " 1 " is considered living above the poverty line, and a score of less than " 1 " is considered to be below the poverty level

Note $N=203$ is the number of participants who enrolled at baseline and who participated in the 3-month follow-up

$H S$ high school, US United States, IPV intimate partner violence, PTSD posttraumatic stress disorder

responded "yes" to "ever exposed" to IPV but "no" to IPV exposure within the preceding 12 months were classified as having "remote" IPV exposure. Lifetime IPV exposure for this study was identified by adding "ever" to the abuse exposure questions.

Non-IPV specific trauma (exposure to non-IPV physical, emotional, or sexual traumatic events) was measured by a 7-item index which assessed childhood experience of emotional or physical abuse by a parent or family member (4 items from the Adverse Childhood Experiences 
Survey-ACE [23], and three items from the Trauma History Questionnaire (THQ) [24]) that identified sexual abuse from someone other than an intimate partner, witnessing household violence prior to age 18 , and parental loss/separation prior to age 18. Positive responses to each item were summed and scores range from 0-7 with higher scores reflecting higher exposure to early trauma.

Social support was measured during pregnancy with a modified version of the Medical Outcomes Study Social Support Survey that included 4-items assessing instrumental support (e.g., receiving transportation favors) and emotional support (e.g., having someone to talk to) from either formal or informal sources [25]. Scores range from 9 to 45 , with higher scores indicating increased levels of social support. For this sample, reliability was .87 for participants interviewed in English and .87 for participants interviewed in Spanish.

Depressive symptoms were measured during pregnancy with the Beck Depression Inventory Fast Screen (BDI-FS) for Medical Patients [26, 27]. The BDI-FS, which does not assess somatic symptoms, is comprised of 7 items from the BDI-II and was developed to screen for depression in medical settings. Items are rated on a 4-point scale from 0 to 3 and total scores range from 0 to 21. Although a cut-off score was not used in the present study, a recommended cut-off score of $>3$ has been reported to yield a sensitivity of $>0.90$ and specificity of $>0.85$ in detecting major depressive disorders [28]. For this sample, alpha reliability was high $(\dot{\alpha}=.85)$.

Post-traumatic stress disorder (PTSD) symptoms were assessed during pregnancy with the 17-item PTSD Checklist-Civilian Version (PCL-C), which measures the degree of symptoms experienced in the last month, as outlined by the DSM-IV-TR [29]. Respondents were asked to rate the level of distress on PTSD symptoms using a 5-point scale ( $1=$ not at all, $5=$ extremely). A score of 44 or higher is associated with a probable diagnosis of PTSD [30, 31]. The scale is diagnostically sensitive $(.82)$ and has high reliability $(\alpha=.92)$ [31]. In the present study, alpha reliability was .93 for participants interviewed in English and .97 for participants interviewed in Spanish.

Perceived stress was assessed during pregnancy with the four-item version of the Perceived Stress Scale (PSS), a global measure of perceived stress [32]. Participants were asked to rate perceived ability to handle "personal problems" and "important things in life" on a Likert scale with response options of "never," "almost never," "sometimes," "fairly often," and "very often." Items were totaled for a score ranging from 0 to 16 , with higher scores indicating higher levels of stress. The PSS has demonstrated sound reliability, [32] including among Latinas $(\alpha=.75)[33,34]$. In this sample, alpha reliability was .68 for participants interviewed in English and .74 for participants interviewed in Spanish.

Acculturation was assessed using language of interview (Spanish or English).and number of years lived in the United Statues. Based on post-hoc analysis, foreign-born participants were classified into two groups and compared: (1) those with $<10$ years living in the United States (low acculturation) and (2) those with $>10$ years living the United States (high acculturation).

Three variables were used to assess socioeconomic status: family income, employment status, and educational attainment. A poverty index was computed to assess family income and is the ratio of reported total family income to the federal poverty level, based on the number of persons in the household [35].

Health problems experienced during pregnancy or delivery were assessed post-delivery based on seven selfreport questions from the Risk Assessment and Monitoring System (PRAMS), [36] which has been used with large samples of Latinas. PRAMS, which has been used with large samples of Latinas, is an ongoing state and population based surveillance system supported by the Centers for Disease Control that assesses maternal behaviors and experiences. The questions used in the present study asked participants to answer yes or no the following items about their pregnancy experience: (1) labor pains more than 3 weeks before the baby was due (preterm or early labor); (2) high blood pressure (including pre-eclampsia or oxemia); (3) high blood sugar (diabetes); (4) urinary tract infection; (5) sexually transmitted infection; (6) anemia; and (7) non-vaginal delivery. Positive responses were summed, with high scores indicating more problems.

\section{Outcome Measure}

Perceived Postpartum Health status at 12 weeks postpartum was measured by an overall general health status score (i.e., perceived general health), emotional well-being score (role functioning related to emotional health and mood), and physical health status score (i.e., physical limitations, role functioning, pain, and health status), on the Medical Outcomes Study SF-12 [37-39]. The SF-12 consists of 12 items that assess perceived health and provides a global health status score, an emotional well-being subscale score based on 4 of the 12 items that assess role functioning related to emotional health and mood, and a physical health status subscale score based on 4 items that assess physical limitations of role functioning and pain. The SF-12 global health and two subscales have demonstrated reliability and validity with coefficients ranging from 0.70 to 0.80 and reliability estimates at 0.90 [37, 38]. Responses are scored on a 5-category Likert scale as excellent $=5$ to poor $=1$. Scores for overall health status 
and the emotional well-being and physical health domains range from 0 to 100 , with higher scores reflecting better perceived overall health and functioning [39]. In this sample, alpha reliability of SF-12 items was .87 for participants interviewed in either English or Spanish.

\section{Data Analysis}

All analyses were conducted with the SAS version 9.1 software. Statistical significance for all analyses was set at $P<.05$. Fourteen (as shown on Table 2) potential predictors variables were reduced to seven based on statistically significant bivariate correlations (Pearson product-moment for continuous variables and point biserial for dichotomous) between the predictor variables and the SF-12 global overall health score (Table 2): number of years in the US, pregnancy/labor/delivery problems; nonIPV traumatic events; recent IPV trauma; perceived stress; social support; and PTSD symptoms. Analysis of variance (ANOVA) was used to test differences on outcomes by location of interview (clinic, home, phone), to which no significant differences were detected. Based on significant differences detected in the contrast post-hoc analysis, the categorical variable of being born in the US was broken up into three dimensions based on the number of years living in the US (i.e., US-born, lived in the US $<10$ years, lived in the US $>10$ years) was included in the final model as a predictor.

Table 2 Correlations of prenatal factors with SF-12 overall health status score at 12 weeks postpartum $(n=193)$

\begin{tabular}{lcc}
\hline Variable & Correlation & $P$ value \\
\hline Maternal age & -0.086 & .2237 \\
Unmarried & -0.025 & .7218 \\
Employed & 0.014 & .8466 \\
High school graduate & 0.026 & .7182 \\
Language of interview-Spanish & -0.108 & .1237 \\
Poverty index & -0.038 & .6095 \\
Non-US-born $\leq 10$ years in US & 0.19801 & $.0046^{*}$ \\
Pregnancy problems & -0.183 & $.0088^{*}$ \\
Non-IPV traumatic events & -0.303 & $<.0001^{*}$ \\
IPV exposure (recent) & -0.242 & $.0005^{*}$ \\
Perceived stress & -0.405 & $<.0001^{*}$ \\
Social support & 0.261 & $.0002^{*}$ \\
Depressive symptoms & -0.273 & $.0002^{*}$ \\
PTSD symptoms & -0.259 & $.0002^{*}$ \\
\hline
\end{tabular}

$* P<.05$

$I P V$ Intimate Partner Violence, PTSD Posttraumatic stress disorder

\section{Results}

Of the 203 participants with follow-up data at 12 weeks postpartum, 193 had complete data on the outcome measure, resulting in the final sample size of 193 used in study analysis. Three separate multiple regression models were fitted to identify predictors of perceived maternal overall health status as measured by the SF-12 global health score, emotional well-being, and physical well-being, at 12 weeks postpartum. (Table 3) The seven predictor variables identified in the bivariate analysis were best fit to model I (overall health status) and model II (emotional well-being), with $R^{2}$ of 0.28 and 0.24 respectively, compared to $R^{2}$ of 0.09 for model III (physical status).

\section{Overall Health Status}

Overall health status scores ranged from 57.3 to 119.1 , with the mean score in the high average range $(M=102.3$, $\mathrm{SD}=12.21)$. Lower scores on perceived overall health status were demonstrated among women with a higher number of non-IPV trauma events $(P<.05)$, higher levels of perceived stress during pregnancy $(P<.001)$, and a greater number of pregnancy/labor/delivery problems $(P<.05)$. Foreign-born women residing in the US $<10$ years evidenced higher scores on overall health status $(P<.01)$ (Model I in Table 3).

\section{Emotional Well-Being}

Scores on emotional well-being ranged from 7.8 to 70.3 , with the mean score in the average range $(M=49.01$, $\mathrm{SD}=10.86$ ). Higher levels of social support during pregnancy were associated with higher emotional wellbeing scores at 12 weeks postpartum $(P<.01)$. Conversely, levels of perceived stress during pregnancy $(P<.001)$ were significantly and negatively related to emotional well-being. Higher scores on emotional wellbeing were significantly and positively associated with being foreign-born and having resided in the US $\leq 10$ years $(P<.01)$ (Model II in Table 3).

\section{Physical Health Status}

Scores on the physical health subscale ranged from 12.5 to 67.4, with the mean score in the high average range $(M=53.27, \mathrm{SD}=7.56)$. After controlling for all other variables in the model, greater number of non-IPV traumatic events $(P<.05)$ and greater number of pregnancy/ labor/delivery problems $(P<.05)$ were negatively associated with physical health status at 12 weeks postpartum. However, the seven predictor variables accounted for a very small proportion of the variance in postpartum 
Table 3 Regression analyses predicting overall health status, emotional well-being and physical health status at 12 weeks postpartum $(n=193)$

\begin{tabular}{|c|c|c|c|c|c|c|c|c|c|}
\hline \multirow[t]{2}{*}{ Prenatal predictors } & \multicolumn{3}{|c|}{$\begin{array}{l}\text { Model I } \\
\text { overall health status }\end{array}$} & \multicolumn{3}{|c|}{$\begin{array}{l}\text { Model II } \\
\text { emotional well-being }\end{array}$} & \multicolumn{3}{|c|}{$\begin{array}{l}\text { Model III } \\
\text { physical status }\end{array}$} \\
\hline & $B$ & $S E B$ & $P$ value & $B$ & $S B$ & $P$ value & $B$ & $S B$ & $P$ value \\
\hline Non-United States born $\leq 10 \mathrm{yrs}$ in US & 4.814 & 1.61 & $.003^{*}$ & 4.805 & 1.49 & $.002 *$ & 0.008 & 1.11 & .994 \\
\hline Intimate partner violence (IPV) recent & 0.106 & 2.17 & .961 & 2.615 & 2.01 & .194 & -1.509 & 1.50 & .096 \\
\hline Non-IPV trauma history & -1.268 & 0.557 & $.024 *$ & -0.296 & 0.509 & .561 & -0.972 & 0.381 & $.012 *$ \\
\hline Social support & 0.168 & 0.121 & .167 & 0.286 & 0.110 & $.010^{*}$ & -0.118 & 0.083 & .155 \\
\hline Perceived stress & -1.290 & 0.293 & $<.000^{*}$ & -1.199 & 0.267 & $<.001^{*}$ & -0.090 & 0.200 & .653 \\
\hline Posttraumatic stress disorder score & 0.074 & 0.078 & .342 & 0.076 & 0.071 & .283 & -0.002 & 0.053 & .963 \\
\hline Pregnancy problems & -1.501 & 0.737 & .043 & -0.470 & 0.674 & .477 & -1.021 & 0.505 & $.045^{*}$ \\
\hline$R^{2}$ & 0.28 & & & 0.24 & & & 0.09 & & \\
\hline Adjusted $R^{2}$ & 0.25 & & & 0.21 & & & 0.06 & & \\
\hline
\end{tabular}

$* P<.05$

maternal physical well-being $\left(R^{2}=.09\right)$ (Model III in Table 3).

\section{Discussion}

Overall, this longitudinal study of postpartum Latinas found several psychosocial and socio-demographic factors independently predicted perceived postpartum health status, with differential contributions noted for the three parameters of health status (i.e., overall, emotional wellbeing, physical health). Specifically, five prenatal factors emerged as significant predictors of postpartum health status at the 12-week time period: perceived stress, social support, recent IPV exposure, health problems experienced during pregnancy or delivery, and for foreign-born women, years lived in the United States.

Perceived stress during pregnancy demonstrated a particularly robust influence on general perceived health status and emotional well-being at 12 weeks postpartum. Women reported mean scores of perceived stress during pregnancy in the moderate range of severity, and higher levels of perceived stress were found to be inversely related to decreased health status. The importance of this finding is emphasized by the fact that it held even after controlling for symptoms of PTSD, trauma exposure and social support. As such, this result strengthens those of prior studies documenting that women may be particularly vulnerable to the effects of stress in early pregnancy in relation to postpartum health. After controlling for demographic and medical factors, Glynn and colleagues found that women who experienced an earthquake in the first trimester of pregnancy rated the event as more stressful than those in later pregnancy and that stress in early pregnancy contributed to gestation length [40]. Taken together, these data and others provide compelling evidence that perceived stress during pregnancy should be monitored and should receive increased attention in similar research. This research should more fully explore the source(s) of the perceived stress during early pregnancy and determine whether there are differential contributions by stress type (e.g., health-related, interpersonal) associated with health status.

Consistent with other studies documenting the positive role of social support and health among pregnant Latinas, [33] social support appeared to have a strong and protective influence on emotional well-being in this study. That is, increased levels of social support during pregnancy were significantly and positively related to increased levels of postpartum emotional well-being. However, this association was not substantiated in relation to overall health status or physical health status. In a cross-sectional study on multi-ethnic women in late pregnancy, McKee and colleagues did not find social support to be related to emotional well-being or physical health but it was weakly associated with overall health status [14]. A possible explanation for this unexpected finding in the current study may be attributed to the limited variance of social support in the sample (the majority of the sample reported high levels of social support), along with the broader framework of psychosocial factors in which it was examined. High levels of social support in the sample may also help to explain why recent IPV exposure (i.e., in the previous 12 months) was related only to physical health status; social support may have inoculated participants from the more severe emotional distress associated with IPV exposure. Prior findings using several indicators to assess abuse have found childhood trauma and physical and sexual IPV to be associated with health status and emotional wellbeing but did not control for social support. Therefore, future research should confirm this finding while assessing more fully the type, duration and severity of IPV. 
Problems experienced during pregnancy or delivery have been associated with health status, and in some cases, as far out as 14 years postpartum [41]. In our study, such problems were negatively associated with scores on physical health status. Given that this scale assesses role functioning and women have reported difficulties in role functioning for as long as 12 weeks postpartum, this was not a surprising finding.

Foreign-born women who had lived in the United States $<10$ years had higher scores on overall health status and emotional well-being when compared to US-born women and foreign-born women who had lived in the US $>10$ years. This finding is generally congruent with the premise that the health and well-being of foreign-born Latinas tend to decrease with increased time lived in the US [42]. Likewise, period of residency in the US is frequently used as a proxy for acculturation, which has been found to serve a protective role in some aspects of health among foreign-born Latinas [43]. As the length of time immigrants live in the US increases, their exposure to new stressors (e.g., discrimination) likely increases, which might also help to explain this finding [44].

Symptoms of PTSD were correlated with depressive symptoms and overall health status in this sample but did not emerge as an independent predictor. It is possible that this association was not confirmed because of the relatively low mean scores on both measures assessing symptoms of depression and PTSD reported in this sample. Also, the broad psychosocial context in which these symptoms were examined in this study may have influenced this finding (e.g., sub-clinical distress measured by perceived stress). Given that previous studies have found depressive symptoms during pregnancy to be a robust predictor of perceived health status, this finding should be interpreted with caution and replicated in future studies.

Several caveats to study interpretation deserve mention. First, culture-specific factors (e.g., cultural ties, discrimination), have been documented to moderate the effects of psychosocial distress among Latinas but were not examined in the present study $[33,44]$. Additional studies are needed to examine the possible pathways of these factors. Second, because this was a convenience sample of women who were selected for study enrollment based on their lifetime histories of IPV exposure this is not a representative sample. Nonetheless, one study reported IPV exposure rates among US- and foreign-born Latinas in the range of $21-84 \%$, throughout the lifespan [45]. Third, a sizable portion of the sample was foreign-born $(75 \%)$ and born in Mexico $(53.4 \%)$. While approximately $52 \%$ of Latinas in the US are foreign-born [1] and many Latinas residing in the US are of Mexican descent, it is worth noting that Latinas are not a monolithic group; therefore it is not possible to generalize study findings to women of the broader Latina population
[19, 46]. Future studies should include larger samples comprising of foreign-born Latinas of different ethnic backgrounds to examine sub-ethnic variations. Fourth, the reliance of self-report, especially as it relates to the measurement of health problems experienced during pregnancy and delivery may have been influenced by recall bias. Finally, while the range of socio-demographic and psychosocial factors considered in the current study design reflects one of the most comprehensive to date, not all factors that have been associated with maternal health status, such as infant factors were included [47].

These study limitations not withstanding, confidence in study findings center on the respectable sample size of lowincome Latinas, the longitudinal design and the use of psychometrically sound instruments, strengths rarely seen in similar studies. To aid in promoting optimal health during the postpartum period, these data support clinician screening in early pregnancy. Screening need not be time-consuming or costly. For example, simply asking women or using brief measures (e.g., the number of years a foreign-born woman has resided in the United States, trauma history, stress and social support) during their initial obstetric appointments are both methods that can alert health providers to those women that should be provided with additional resources (e.g., education, support groups) and monitored closely during the postpartum period.. Finally, as health and wellbeing are increasingly emphasized by policy makers, healthcare providers and researchers, these results inform existing frameworks on the health of low-income Latinas and provide a basis for incorporating these factors in future interventions and studies.

Acknowledgments This research was supported in part by the National Institute of Mental Health grants Nos. T32-MH17140; NIMH funded grant Collaborative Center for Trauma and Mental Health Disparities (CCTMHD) 1P50MH073453: 010A1; Department of Health and Human Services/Agency for Health Research and Quality (DHHS/AHRQ), 7 R01 HS11104. The authors thank Lina Palomares for her assistance and the women in this study who generously gave of their time and experiences to make this study possible.

Open Access This article is distributed under the terms of the Creative Commons Attribution Noncommercial License which permits any noncommercial use, distribution, and reproduction in any medium, provided the original author(s) and source are credited.

\section{References}

1. US Census Bureau. (2000). US census 2000. Washington, DC: US Census Bureau.

2. Martin, J. A., Hsiang-Ching, K., Mathews, T. J., et al. (2008). Annual summary of vital statistics: 2006. Pediatrics, 121, 788-801.

3. Wisner, K. L., \& Wheeler, S. B. (1994). Prevention of recurrent postpartum major depression. Hospital \& Community Psychiatry, $45,1191-1195$. 
4. Zayas, L. H., Cunningham, M., McKee, M. D., \& Jankowski, K. R. (2002). Depression and negative life events among pregnant African-American and Hispanic women. Women's Health Issues, 12, 16-22.

5. Smith, K., Downs, B., \& O'Connell, M. (2001). Maternity leave and employment patterns: 1961-1996. Current population reports (pp. 70-79). Washington, DC: US Census Bureau.

6. McGovern, P., Dowd, B., Gjerdingen, D., Dagher, R., Ukestad, L., McCaffrey, D., et al. (2007). Mothers health and work-related factors at 11 weeks postpartum. Annals of Family Medicine, 5, 519-527.

7. Cheng, C. Y., Fowles, E. R., \& Walker, L. O. (2006). Postpartum maternal health care in the United States: a critical review. The Journal of Perinatal Education, 15(3), 34-42.

8. McGlade, M. S., Saha, S., \& Dahlstrom, M. E. (2004). The Latina paradox: an opportunity for restructuring prenatal care delivery. American Journal of Public Health, 94(12), 2062-2065.

9. United Nations. (2008). The millennium development goals report 2008. New York: United Nations Department of Economic and Social Affairs.

10. Haas, J. S., Jackson, R. A., Fuentes-Afflick, E., et al. (2005). Changes in the health status of women during and after pregnancy. Journal of General Internal Medicine, 20, 45-51.

11. McCormick, M. C., Brooks-Gunn, J., Holmes, J. H., Wallace, C. Y., \& Heagarty, M. C. (1992). Maternal health status in the year after delivery among low-income women. Journal of Women's Health, 1(3), 225-230.

12. Nicholson, W. K., Setse, R., Hill-Briggs, F., Cooper, L. A., Strobino, D., \& Powe, N. R. (2006). Depressive symptoms and health-related quality of life in early pregnancy. Obstetrics and Gynecology, 107, 798-806.

13. Setse, R., Grogran, R., Pham, L., et al. (2008). Longitudinal study of depressive symptoms and health-related quality of life during pregnancy and after delivery: the health status in pregnancy (HIP) study. Maternal and Child Health Journal, 13(5), 577-587.

14. McKee, M. D., Cunningham, M., Jankowski, K. R. B., \& Zayas, L. (2001). Health-related functional status in pregnancy: relationship to depression and social support in a multi-ethnic population. Obstetrics and Gynecology, 9, 988-993.

15. Idler, E. L., \& Benyamini, Y. (1997). Self-rated health and mortality: a review of twenty-seven community studies. Journal of Health and Social Behavior, 38, 21-37.

16. Hennessy, C. H., Moriarty, D. G., Zack, M. M., Scherr, P. A., \& Brackbill, R. (1994). Measuring health-related quality of life for public health surveillance. Public Health Reports, 109(5), 665-672.

17. Bonomi, A. E., Anderson, M. L., Rivara, F. P., \& Thompson, R. S. (2007). Health outcomes in women with physical and sexual intimate partner violence exposure. Journal of Women's Health, 16(7), 987-997.

18. Bonomi, A. E., Cannon, E. A., Anderson, M. L., Rivara, F. P., \& Thompson, R. S. (2008). Association between self-reported health and physical and/or sexual abuse experienced before age 18. Child Abuse and Neglect, 32(7), 693-701.

19. Jerant, A., Arellanes, R., \& Franks, P. (2008). Health status among US Hispanics: ethnic variation, nativity, and language moderation. Medical Care, 46(7), 709-717.

20. Bonomi, A. E., Anderson, M. L., Cannon, E. A., \& Rodriguez, M. A. (2009). Intimate partner violence in Latina and non-Latina women. American Journal of Preventive Medicine, 36(1), 43-48.

21. Rodriguez, M. A., Heilemann, M. V., Fielder, E., Ang, A., Nevarez, F., \& Mangione, C. M. (2008). Intimate partner violence, depression, and PTSD among pregnant Latina women. Annals of Family Medicine, 6, 44-52.

22. Soeken, K., McFarlane, J., Parker, B., \& Lominack, M. C. (1998). The abuse assessment screen: a clinical instrument to measure frequency, severity, and perpetrator of abuse against women. In J. Campbell (Ed.), Empowering survivors of abuse: health care for battered women and their children (pp. 195-203). Thousand Oaks: Sage Publications.

23. Felitti, V. J., Anda, R. F., Nordenberg, D., et al. (1998). Relationship of childhood abuse and household dysfunction to many of the leading causes of death in adults. The adverse childhood experiences (ACE) study. American Journal of Preventive Medicine, 14(4), 245-258.

24. Green, B. L. (1996). Trauma history questionnaire. In B. H. Stamm (Ed.), Measurement of stress, self-report trauma and adaptation (pp. 366-368). Lutherville, MD: Sidran Press.

25. Sherbourne, C. D., \& Stewart, A. L. (1991). The MOS social support survey. Social Science and Medicine, 32, 705-714.

26. Beck, A. T., Steer, R. A., \& Brown, G. K. (2000). BDI-Fast screen for medical patients: manual. San Antonio: The Psychological Corporation.

27. Steer, R. A., Cavalieri, T. A., Leonard, D. M., \& Beck, A. T. (1999). Use of the Beck depression inventory for primary care to screen for major depression disorders. General Hospital Psychiatry, 21, 106-111.

28. Beck, A. T., Steer, R. A., Roberta, B., Carman, A., \& Kabat, M. (1997). The use of the beck anxiety and depression inventories for primary care with medical outpatients. Assessment, 4(3), 211-219.

29. Walker, E. A., Newman, E., Dobie, D. J., Ciechanowski, P., \& Katon, W. J. (2002). Validation of the PTSD checklist in an HMO sample of women. General Hospital Psychiatry, 24(6), 375-380.

30. Cordova, M. J., Andrykowski, M. A., Kenady, D. E., McGrath, P. A., Sloan, D. A., \& Redd, W. H. (1995). Frequency, correlates of posttraumatic stress disorder-like symptoms after treatment for breast cancer. Journal of Consulting and Clinical Psychology, 63, 981-986.

31. Smith, M. Y., Egert, J., Winkel, G., \& Jacobson, J. (2002). The impact of PTSD on pain experiences in persons with HIV/AIDS. Pain, 98, 9-17.

32. Cohen, S., Kamarck, T., \& Mermelstein, R. (1983). A global measure of perceived stress. Journal of Health and Social Behavior, 24, 385-396.

33. Campos, B., Dunkel-Schetter, C., Abdou, C. M., Hobel, C. J., Glynn, L. M., \& Sandman, C. A. (2008). Familialism, social support and stress: positive implications for pregnant Latinas. Cultural Diversity \& Ethnic Minority Psychology, 14(2), 155-162.

34. Zambrana, R. E., Scrimshaw, S. C. M., Collins, N., \& DunkelSchetter, C. (1997). Prenatal health behaviors and psychosocial risk factors in pregnant women of Mexican origin: the role of acculturation. American Journal of Public Health, 87, 1022-1026.

35. US Department of Health and Human Services. (2006). The 2004 HHS Poverty Guidelines. http://aspe.hhs.gov/poverty/04poverty. shtml. Accessed June 2, 2006.

36. Gilbert, B. C., Shulman, H. B., Fischer, L. A., \& Rogers, M. M. (1999). The pregnancy risk assessment monitoring system (PRAMS): methods and 1996 responses rates from 11 states. Maternal and Child Health Journal, 3(4), 199-209.

37. Ware, J. E. (1986). The assessment of health status. In L. H. Aiken (Ed.), Applications of social science to clinical medicine and health policy. Piscataway, New Jersey: Rutgers University Press.

38. Ware, J. E., Kosinki, M., \& Susan, K. (1996). A 12-item shortform health survey: construction of scales and preliminary tests of reliability and validity. Medical Care, 32(3), 220-233.

39. Ware, J. E., Kosinski, M., \& Keller, S. D. (1998). SF- 12: how to score the SF-12 physical and mental health summary scales (3rd ed.). Lincoln, RI: QualityMetric.

40. Glynn, L. M., Wadhwa, P. D., Dunkel-Schetter, C., Chicz-DeMet, A., \& Sandman, C. A. (2001). When stress happens matters: effects 
of earthquake timing on stress responsivity in pregnancy. American Journal of Obstetrics and Gynecology, 184, 637-642.

41. Khatun, M., Clavarino, A. M., Callaway, M., Alati, R., Najman, J. M., Williams, G., et al. (2009). Common symptoms during pregnancy to predict depression and health status 14 years postpartum. International Journal of Gynaecology and Obstetrics, 104(3), 214-217.

42. Abraído-Lanza, A. F., Dohrenwend, B. P., Ng-Mak, D. S., \& Turner, J. B. (1999). The Latino mortality paradox: a test of the "salmon bias" and healthy migrant hypotheses. American Journal of Public Health, 89, 1543-1548.

43. Davila, M., McFall, S. L., \& Cheng, D. (2009). Acculturation and depressive symptoms among pregnant and postpartum Latinas. Maternal and Child Health Journal, 13(3), 318-325.

44. Gee, G. C., Ryan, A., Laflamme, D. J., \& Holt, J. (2006). Self-reported discrimination and mental health status among
African descendants, Mexican Americans, and other Latinos in the New Hampshire REACH 2010 Initiative: The added dimension of immigration. American Journal of Public Health, 96(10), $1821-1828$.

45. Hazen, A. L., \& Soriano, F. I. (2007). Experiences with intimate partner violence among Latina women. Violence Against Women, 13(6), 562-582.

46. Amaro, H., \& De la Torre, A. (2002). Public health needs and scientific opportunities in research on Latinas. American Journal of Public Health, 92(4), 525-529.

47. Schytt, E., \& Waldenstrom, U. (2007). Risk factors for poor selfrated health in women at 2 months and 1 year after childbirth. Journal of Women's Health, 16(3), 390-405. 